\title{
Acacetin as a Potential Protective Compound against Cardiovascular Diseases
}

\author{
Chunxuan Wu, Jianhua Yan $\mathbb{D}$, and Wei Li \\ Department of Cardiology, Xinhua Hospital, School of Medicine, Shanghai Jiao Tong University, Shanghai, China \\ Correspondence should be addressed to Jianhua Yan; yanjianhua@xinhuamed.com.cn and Wei Li; liwei@xinhuamed.com.cn
}

Received 30 October 2021; Accepted 8 February 2022; Published 2 March 2022

Academic Editor: Yong Wang

Copyright ( $) 2022$ Chunxuan Wu et al. This is an open access article distributed under the Creative Commons Attribution License, which permits unrestricted use, distribution, and reproduction in any medium, provided the original work is properly cited.

Acacetin (5,7-dihydroxy-4'-methoxyflavone) is the major bioactive component of the traditional Chinese medicine "Snow lotus". As a natural flavonoid compound, it has been shown to have good pharmacological effects such as anti-inflammatory, anticancer, and anti-obesity. Among them, its prominent role in cardiovascular diseases (CVD) has received extensive attention from scholars in recent years. In this review, the protective effects of acacetin on a variety of cardiovascular diseases, as well as the existing problems and prospects, are discussed and summarized. This review also highlights the great potential of acacetin, a naturalderived Chinese medicine, as a cardiovascular agent candidate.

\section{Introduction}

Cardiovascular disease (CVD), including coronary heart disease, hypertension, myocardial infarction, and heart failure, is not only the main cause of death worldwide but also a major public health problem $[1,2]$. Although many great achievements have been made in the prevention and treatment of CVD in the past decades, the morbidity and mortality of CVD continue to increase due to population growth and ageing [3]. Therefore, it is of great significance to develop alternative or complementary therapies for cardiovascular diseases.

Traditional Chinese medicine (TCM) has a history of more than 2,000 years. It is not only popular in China but also highly recognized in many countries in the world [4]. In recent years, more and more TCMs have been proved to be potential candidates for the treatment of cardiovascular disease, and their mechanisms is becoming clearer, such as berberine, curcumin, Wenxin Keli, resveratrol, and acacetin [5-8].

Acacetin (5,7-dihydroxy-4'-methoxyflavone), one of the flavones of flavonoids, is a monomethoxy flavonoid, which exists in a variety of plants in free form or glycoside form, including Robinia pseudoacacia, Chrysanthemum indicum, and other species $[9,10]$ (Figure 1). Acacetin has attracted wide attention from the scientific community because of its various pharmacological activities, including anti-inflammatory, anticancer, anti-obesity, anti-diabetic, neuroprotective, and cardioprotective effects $[9,11]$. Among them, the application prospect of acacetin in the CVD field is the most noteworthy (Figure 2). It has good curative effects on CVD with almost no toxic reactions, such as atrial fibrillation, ischemia/reperfusion injury, atherosclerosis, doxorubicin cardiomyopathy, and cardiac senescence.

This review focuses on the protective effects of acacetin on CVD, as well as the main signal pathways regulated under different pathophysiological conditions in CVD. This review also emphasizes the current problems and challenges related to acacetin in the treatment of CVD, which need further experimental exploration and make it a candidate for the prevention and treatment of CVD.

\section{Pharmacological Effects on Cardiovascular Diseases}

\subsection{Cardiac Arrhythmias}

2.1.1. Atrial Fibrillation. Atrial fibrillation (AF) is one of the most common cardiac arrhythmias in clinical practice, and its incidence increases with age [12]. Complications of 


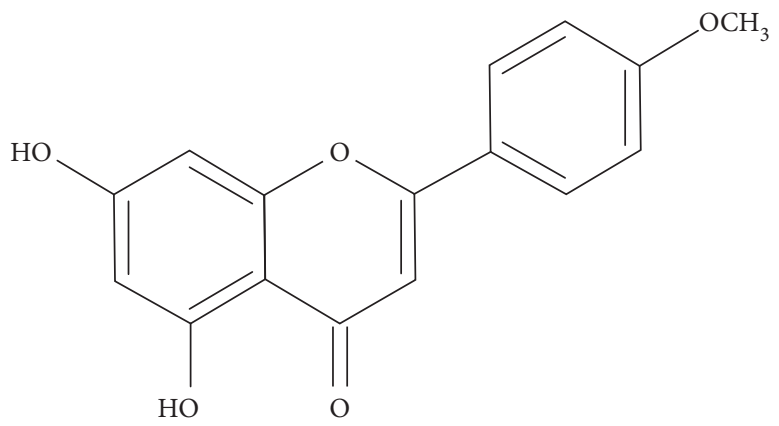

FIgURE 1: Chemical structure of acacetin.

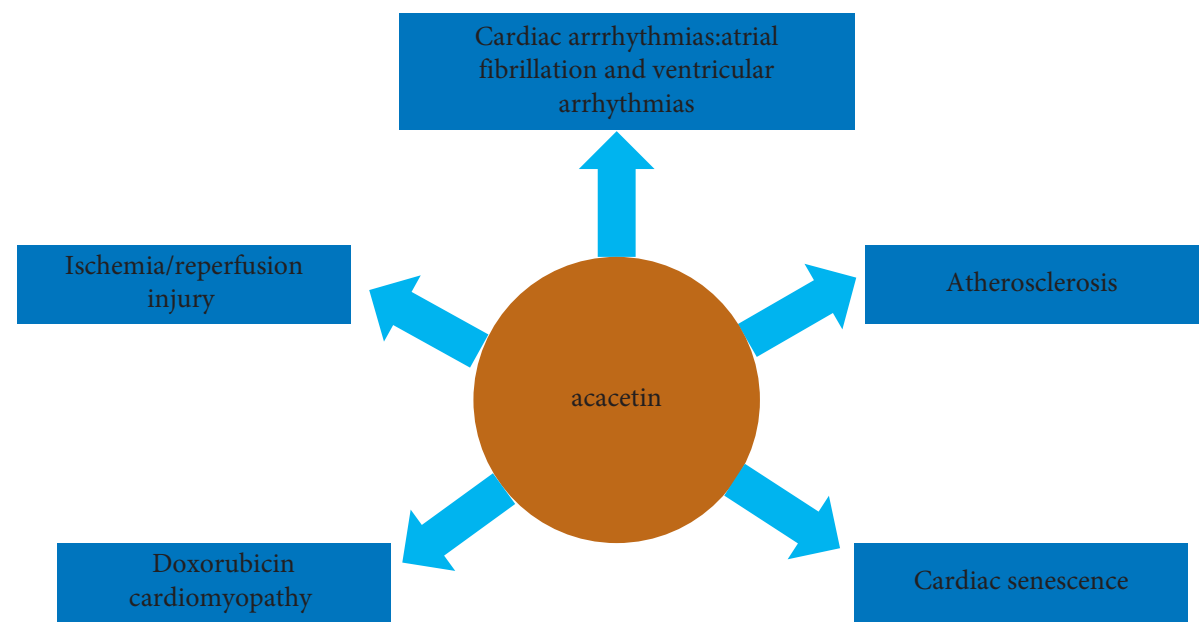

FIgURe 2: Pharmacological properties of acacetin in cardiovascular diseases.

cardiac and extra-cardiac caused by AF significantly increase morbidity and mortality $[13,14]$. In the past two decades, catheter ablation has become the mainstream strategy of rhythm control recommended by clinical guidelines, but antiarrhythmic drugs are also an indispensable part of controlling AF and its recurrence $[15,16]$. The development of atrial-selective antiarrhythmic drugs is important for the treatment of AF, because if the drug acts on the ventricles, it may prolong the action potential duration (APD) of the ventricles and even cause the appearance of lethal ventricular arrhythmias such as torsade de pointes (Tdp) $[17,18]$.

Recently, Li et al. have demonstrated that acacetin can preferentially inhibit $I_{\text {Kur }}$ (ultra-rapidly activated delayed rectifier potassium current), $I_{\text {to }}$ (transient outward potassium current), $I_{\mathrm{KACh}}$ (acetylcholine activated potassium current), and SKca (small conductance $\mathrm{Ca}^{2+}$ activated potassium channels current), while it had no effect on the $\mathrm{Na}^{+}$current, L-type $\mathrm{Ca}^{2+}$ current, or inwardrectifier $\mathrm{K}^{+}$current in guinea pig cardiac myocytes $[19,20]$. Among them, $I_{\text {kur }}$ is specifically expressed in the human atrium but not in the ventricle, which is an ideal target for atrial-selective antiarrhythmic drugs [21, 22]. In addition, previous studies have shown that selective $I_{\mathrm{KACh}}$ inhibitors prevent AF induced by vagal stimulation $[23,24]$. Intraduodenal administration of acacetin can effectively prevent AF in anesthetized dogs by increasing the APD and prolonging the effective refractory period (ERP) in atrial myocytes. More importantly, compared with sotalol, acacetin can effectively prevent AF without QT interval (QTc) prolongation. These results suggest that acacetin is an atrium-selective agent and has great potential to treat AF. Considering that acacetin has poor water solubility, it can only be taken orally and cannot be used for intravenous injection to terminate AF. In 2016, a water-cooled phosphate acacetin prodrug which can be quickly converted into the active form acacetin was designed [25]. The study shows that the synthesized acacetin prodrug is highly water-soluble and safe. Furthermore, it can also effectively terminate experimental AF in beagle dogs.

The molecular determinants of acacetin's ability to block $I_{\text {to }}$ and $I_{\text {kur }}$ were further investigated $[26,27]$. The results show that acacetin has a use-dependent and frequencydependent blocking effect on hKv4.3 (coding $I_{\text {to }}$ ) and hKv1.5 (coding $I_{\text {kur }}$ ) channels. Acacetin, as an antagonist of the hKv4.3 channel, interacts with T366A and T367A located in the P-loop helix and V392A, I395A, and V399A located in the S6-segment [26]. In addition, acacetin, as a hKv1.5 channel antagonist, interacts with V505A, I508A, and V512A located in the S6 domain [27]. 
In addition, the combination of acacetin and sodium channel blockers has also been shown to be a promising strategy for the treatment of AF [28].

2.1.2. Ventricular Arrhythmias. J wave syndromes, mainly including Brugada syndrome $(\mathrm{BrS})$ and early repolarization syndrome (ERS), are characterized by an abnormal transition point between the terminal part of the QRS complex and the ST segment, that is, the $J$ point in the electrocardiogram. It is well known that $J$ wave syndromes are associated with serious ventricular tachycardia (VT), ventricular fibrillation (VF), and other life-threatening events [29, 30]. Implantable cardioverter defibrillator (ICD) is recommended as the preferred therapy for the prevention of sudden cardiac death in high-risk patients with $J$ wave syndrome. But the drugs for the diseases are extremely limited. Quinidine, an Ito inhibitor, is one of the few drugs that are effective in treating $J$ wave syndromes [31]. More recently, Di Diego et al. have shown that acacetin can noticeably improve the electrocardiogram and arrhythmia performance in both the BrS and ERS experimental models by reducing $\mathrm{I}_{\text {to }}$ density, action potential notch, and $J$ wave area [32].

2.2. Ischemia/Reperfusion Injury and Myocardial Infarction. In 2014, Yang et al. for the first time demonstrated that acacetin in vitro can reduce the hypoxia/reoxygenation injury to the cardiac myocardium and has a protective effect on the myocardium by reducing lipid peroxidation and enhancing the antioxidant capacity [33]. In this study, acacetin can significantly reduce the content of malondialdehyde (MDA), which is usually used to evaluate the oxidative damage of cells in the medium during hypoxia/ reoxygenation injury. Subsequently, an acacetin phosphate prodrug was applied to the in vivo experiments of ischemia/ reperfusion injury, and the studies showed that acacetin inhibited the apoptosis of myocardial cells by preventing the reduction of antioxidants such as SOD-2 and thioredoxin and reducing the release of inflammatory cytokines such as TLR4, IL-6, and TNF $\alpha$, thus playing a protective role on the myocardium after myocardial infarction (MI) [34]. Further studies have confirmed that the antioxidant, anti-inflammatory, and antiapoptotic effects of acacetin on cells are mediated by AMPK-mediated Nrf2 activation [35]. Interestingly, a recent study demonstrated that autophagy is involved in the process of alleviation of myocardial hypoxia/ reoxygenation injury by acacetin; that is, acacetin enhanced autophagy through activating the PI3K/Akt/mTOR pathway [36].

Myocardial infarction is one of the most prominent cardiovascular diseases, contributing significantly to death. Cardiac remodeling is an important process after myocardial infarction, which is closely related to the prognosis of the disease. Recent experiments have shown that acacetin can inhibit myocardial remodeling such as myocardial hypertrophy and fibrosis, reduce myocardial infarct size, and enhance left ventricular function after myocardial infarction [37]. The protective effect of acacetin on cardiac remodeling after myocardial infarction was attributed to its inhibition of MAPK and PI3K/Akt signaling pathways, which play important roles in myocardial remodeling after myocardial infarction [38].

2.3. Atherosclerosis. Atherosclerosis used to be regarded as a chronic inflammatory disease related to dyslipidaemia, but more and more evidence shows that oxidative stress is also involved [39]. Considering the powerful antioxidant and anti-inflammatory ability of acacetin, Yao et al. studied the potential of acacetin's application in atherosclerosis [40]. On the one hand, acacetin enhances the antioxidant defense of cells through the MsrA-Nrf2/Keap1 pathway and significantly reduces apoptosis. On the other hand, it can accelerate lipid metabolism in the liver and circulation, and has antiinflammatory effects. In fact, statins, the widely used antihyperlipidemia drugs, have been demonstrated to alleviate oxidative stress by regulating the redox system, which may further increase the possibility of clinical use of acacetin in preventing and treating atherosclerosis-related CVD [41].

2.4. Doxorubicin Cardiomyopathy. Doxorubicin is an anthracycline antibiotic, which is widely used as an anticancer drug in clinics [42]. However, the most significant and harmful side effect of doxorubicin is the increasing cardiovascular toxicity events, including proarrhythmia, hypotension, and congestive heart failure [43]. Dexrazoxane is the only drug to be approved by the FDA to deal with cardiomyopathy caused by doxorubicin, but it has been demonstrated to increase the risk of secondary malignancy [44]. It is worth noting that reactive oxygen species (ROS) is considered to be the most important mechanism of doxorubicin cardiomyopathy [45]. Acacetin has been shown to reduce apoptosis and ROS production and enhance endogenous antioxidants (SOD1, SOD2, and HO-1) via Sirt1dependent activation of AMPK/Nrf2 signals, thus inhibiting myocardial toxicity and cardiac dysfunction caused by doxorubicin [46]. Therefore, acacetin may be a very promising candidate for the treatment of doxorubicin cardiomyopathy in the future.

2.5. Cardiac Senescence. Ageing is considered an independent risk factor for cardiovascular disease, and cardiac senescence is closely related to the occurrence and development of cardiomyopathy in the elderly [47]. Since population ageing is a current situation in many countries, relevant anticardiac ageing treatments are urgently needed. Among them, the change in mitochondrial metabolism is considered as an important mechanism of myocardial ageing [48]. Interestingly, acacetin can significantly improve the cardiac function in the D-galactose-induced cardiac senescence model, reduce senescence markers such as P53 and P21, increase autophagy protein, and increase mitochondrial autophagy via Sirt1-mediated activation of the Sirt6/AMPK signaling pathway [49]. This study suggests that acacetin may be a promising drug candidate for treating ageing-related cardiovascular disorders. 


\section{Problems and Future Prospects}

Although acacetin is a safe and promising candidate for the treatment of CVD, there are still many problems to be resolved in the future. One of the most difficult problems is the poor solubility and rapid metabolism of acacetin in vivo, which will be a major obstacle to the clinical development and utilization of acacetin in the future [50]. In 2016, a study showed us a way to improve acacetin's water-solubility by designing prodrugs to treat AF [25]. This method of designing prodrugs to change the properties of the drug and overcome the shortcomings of the drug plays a role in the initial development of the drug and the various stages of preclinical trials $[51,52]$. However, there are few studies on the development of acacetin prodrugs.

On the other hand, although the pathophysiological conditions of various CVDs are simulated in animals, there are few applications of uniform animal experimental models that are recognized around the world. The in-depth research of acacetin in this area in the future will be an important milestone in promoting preclinical experiments.

In addition, the clinical pharmacological information of acacetin, which is considered to be an important part of drugs, such as absorption, metabolism, and toxicity, is not sufficient at present $[53,54]$. Therefore, all of these need to be further explored and improved upon, so as to confirm the safety and effectiveness of acacetin in human body. Overall, there is still a great distance to the routine clinical application of acacetin. However, it cannot be denied that acacetin is a promising natural candidate for cardiovascular drugs.

\section{Conclusion}

In general, this review emphasizes the great potential of acacetin as a traditional Chinese medicine on cardiovascular disease and summarizes the remarkable effects and related possible mechanisms of acacetin against atrial fibrillation, ischemia-reperfusion injury, atherosclerosis, doxorubicin cardiomyopathy, and cardiac senescence. Further studies are warranted to resolve the current problems of acacetin, such as poor solubility and rapid metabolism in vivo, which may help prevent and treat cardiovascular diseases in the future.

\section{Abbreviations}

AF: Atrial fibrillation

APD: Action potential duration

BrS: Brugada

CVD: Cardiovascular diseases

ERP: Effective refractory period

ERS: Early repolarization syndromes

$\mathrm{I}_{\mathrm{KACh}}$ : Acetylcholine-activated potassium current

$\mathrm{I}_{\text {Kur: }} \quad$ Ultra-rapidly activated delayed rectifier potassium current

$\mathrm{I}_{\mathrm{to}}$ : Transient outward potassium current

MI: Myocardial infarction

QTc: QT interval

ROS: Reactive oxygen species
SKca: Small conductance $\mathrm{Ca}^{2+}$-activated potassium channels current

TCM: Traditional Chinese medicine

Tdp: Torsade de pointes

VT: Ventricular tachycardia

VF: Ventricular fibrillation.

\section{Conflicts of Interest}

The authors declare that they do not have any conflicts of interest.

\section{References}

[1] A. Timmis, N. Townsend, C. Gale et al., "European society of cardiology: cardiovascular disease statistics 2017," European Heart Journal, vol. 39, pp. 508-579, 2018.

[2] E. J. Benjamin, S. S. Virani, C. W. Callaway et al., "Heart disease and stroke statistics-2018 update: a report from the American heart association," Circulation, vol. 137, pp. e67-e492, 2018.

[3] G. A. Roth, M. H. Forouzanfar, A. E. Moran et al., "Demographic and epidemiologic drivers of global cardiovascular mortality," New England Journal of Medicine, vol. 372, no. 14, pp. 1333-1341, 2015.

[4] A. Tachjian, V. Maria, and A. Jahangir, "Use of herbal products and potential interactions in patients with cardiovascular diseases," Journal of the American College of Cardiology, vol. 55, no. 6, pp. 515-525, 2010.

[5] X. Feng, A. Sureda, S. Jafari et al., "Berberine in cardiovascular and metabolic diseases: from mechanisms to therapeutics," Theranostics, vol. 9, no. 7, pp. 1923-1951, 2019.

[6] S. Jiang, J. Han, T. Li et al., "Curcumin as a potential protective compound against cardiac diseases," Pharmacological Research, vol. 119, pp. 373-383, 2017.

[7] G. Tian, Y. Sun, S. Liu et al., "Therapeutic effects of wenxin keli in cardiovascular diseases: an experimental and mechanism overview," Frontiers in Pharmacology, vol. 9, Article ID 1005, 2018.

[8] E. Chong, S.-L. Chang, Y.-W. Hsiao et al., "Resveratrol, a red wine antioxidant, reduces atrial fibrillation susceptibility in the failing heart by PI3K/AKT/eNOS signaling pathway activation," Heart Rhythm, vol. 12, no. 5, pp. 1046-1056, 2015.

[9] H. Abdallah, M. Farag, S. Osman et al., "Isolation of major phenolics fromLaunaea spinosaand their protective effect on HepG2 cells damaged witht-BHP," Pharmaceutical Biology, vol. 54, no. 3, pp. 536-541, 2016.

[10] R. B. Semwal, D. K. Semwal, S. Combrinck, J. Trill, S. Gibbons, and A. Viljoen, "Acacetin-A simple flavone exhibiting diverse pharmacological activities," Phytochemistry Letters, vol. 32, pp. 56-65, 2019.

[11] S. Singh, P. Gupta, A. Meena, and S. Luqman, "Acacetin, a flavone with diverse therapeutic potential in cancer, inflammation, infections and other metabolic disorders," Food and Chemical Toxicology, vol. 145, Article ID 111708, 2020.

[12] S. Nattel and D. Dobrev, "Controversies about atrial fibrillation mechanisms," Circulation Research, vol. 120, no. 9, pp. 1396-1398, 2017.

[13] T. J. Wang, M. G. Larson, D. Levy et al., "Temporal relations of atrial fibrillation and congestive heart failure and their joint influence on mortality," Circulation, vol. 107, no. 23, pp. 2920-2925, 2003.

[14] N. Jost, Z. Kohajda, A. A. Kristof et al., "Atrial remodeling and novel pharmacological strategies for antiarrhythmic therapy 
in atrial fibrillation," Current Medicinal Chemistry, vol. 18, no. 24, pp. 3675-3694, 2011.

[15] R. S. Wijesurendra and B. Casadei, "Mechanisms of atrial fibrillation," Heart, vol. 105, no. 24, pp. 1860-1867, 2019.

[16] M. K. Chung, M. Refaat, W. K. Shen et al., "Atrial fibrillation: JACC council perspectives," Journal of the American College of Cardiology, vol. 75, pp. 1689-1713, 2020.

[17] A. Choudhury and G. Y. Lip, "Antiarrhythmic drugs in atrial fibrillation: an overview of new agents, their mechanisms of action and potential clinical utility," Expert Opinion on Investigational Drugs, vol. 13, no. 7, pp. 841-855, 2004.

[18] A. Burashnikov, J. M. Di Diego, A. C. Zygmunt, L. Belardinelli, and C. Antzelevitch, "Atrium-selective sodium channel block as a strategy for suppression of atrial fibrillation," Circulation, vol. 116, no. 13, pp. 1449-1457, 2007.

[19] G.-R. Li, H.-B. Wang, G.-W. Qin et al., “Acacetin, a natural flavone, selectively inhibits human atrial repolarization potassium currents and prevents atrial fibrillation in dogs," Circulation, vol. 117, no. 19, pp. 2449-2457, 2008.

[20] K.-H. Chen, H. Liu, H.-Y. Sun et al., "The natural flavone acacetin blocks small conductance Ca2+-activated $\mathrm{K}+$ channels stably expressed in HEK 293 cells," Frontiers in Pharmacology, vol. 8, p. 716, 2017.

[21] J. Tamargo, R. Caballero, R. Gómez, and E. Delpón, "IKur/ Kv1.5 channel blockers for the treatment of atrial fibrillation," Expert Opinion on Investigational Drugs, vol. 18, no. 4, pp. 399-416, 2009.

[22] G.-R. Li, J. Feng, L. Yue, M. Carrier, and S. Nattel, "Evidence for two components of delayed rectifier $\mathrm{K}+$ current in human ventricular myocytes," Circulation Research, vol. 78, no. 4, pp. 689-696, 1996.

[23] D. Li, H. Sun, and P. Levesque, "Antiarrhythmic drug therapy for atrial fibrillation: focus on atrial selectivity and safety," Cardiovascular and Hematological Agents in Medicinal Chemistry, vol. 7, no. 1, pp. 64-75, 2009.

[24] K. B. Walsh, "Targeting cardiac potassium channels for stateof-the-art drug discovery," Expert Opinion on Drug Discovery, vol. 10, no. 2, pp. 157-169, 2015.

[25] H. Liu, Y.-J. Wang, L. Yang et al., "Synthesis of a highly watersoluble acacetin prodrug for treating experimental atrial fibrillation in beagle dogs," Scientific Reports, vol. 6, no. 1, Article ID 25743, 2016.

[26] H.-J. Wu, H.-Y. Sun, W. Wu, Y.-H. Zhang, G.-W. Qin, and G.-R. Li, "Properties and molecular determinants of the natural flavone acacetin for blocking hKv4.3 channels," PLoS One, vol. 8, no. 3, Article ID e57864, 2013.

[27] H.-J. Wu, W. Wu, H.-Y. Sun et al., "Acacetin causes a frequency- and use-dependent blockade of hKv1.5 channels by binding to the S6 domain," Journal of Molecular and Cellular Cardiology, vol. 51, no. 6, pp. 966-973, 2011.

[28] H. Ni, D. G. Whittaker, W. Wang, W. R. Giles, S. M. Narayan, and H. Zhang, "Synergistic anti-arrhythmic effects in human atria with combined use of sodium blockers and acacetin," Frontiers in Physiology, vol. 8, p. 946, 2017.

[29] C. Antzelevitch and G.-X. Yan, "J-wave syndromes: Brugada and early repolarization syndromes," Heart Rhythm, vol. 12, no. 8, pp. 1852-1866, 2015.

[30] J. Sieira, G. Dendramis, and P. Brugada, "Pathogenesis and management of Brugada syndrome," Nature Reviews Cardiology, vol. 13, no. 12, pp. 744-756, 2016.

[31] C. Antzelevitch, G.-X. Yan, M. J. Ackerman et al., "J-Wave syndromes expert consensus conference report: emerging concepts and gaps in knowledge," Heart Rhythm, vol. 13, no. 10, pp. e295-e324, 2016.
[32] J. M. Di Diego, B. Patocskai, H. Barajas-Martinez et al., "Acacetin suppresses the electrocardiographic and arrhythmic manifestations of the J wave syndromes," PLoS One, vol. 15, no. 11, Article ID e0242747, 2020.

[33] W.-J. Yang, C. Liu, Z.-Y. Gu et al., "Protective effects of acacetin isolated from Ziziphora clinopodioides Lam. (Xintahua) on neonatal rat cardiomyocytes," Chinese Medicine, vol. 9, no. 1, p. 28, 2014.

[34] H. Liu, L. Yang, H.-J. Wu et al., "Water-soluble acacetin prodrug confers significant cardioprotection against ischemia/reperfusion injury," Scientific Reports, vol. 6, no. 1, Article ID 36435, 2016.

[35] W.-Y. Wu, Y.-D. Li, Y.-K. Cui et al., "The natural flavone acacetin confers cardiomyocyte protection against hypoxia/ reoxygenation injury via AMPK-mediated activation of $\mathrm{Nrf} 2$ signaling pathway," Frontiers in Pharmacology, vol. 9, p. 497, 2018.

[36] C. Liu, M. Zhang, S. Ye et al., "Acacetin protects myocardial cells against hypoxia-reoxygenation injury through activation of autophagy," Journal of Immunology Research, vol. 2021, Article ID 9979843, 12 pages, 2021.

[37] W. Chang, Q.-Q. Wu, Y. Xiao et al., “Acacetin protects against cardiac remodeling after myocardial infarction by mediating MAPK and PI3K/Akt signal pathway," Journal of Pharmacological Sciences, vol. 135, no. 4, pp. 156-163, 2017.

[38] A. J. Muslin, "MAPK signalling in cardiovascular health and disease: molecular mechanisms and therapeutic targets," Clinical Science, vol. 115, no. 7, pp. 203-218, 2008.

[39] P. Libby, P. M. Ridker, and G. K. Hansson, "Progress and challenges in translating the biology of atherosclerosis," Nature, vol. 473, no. 7347, pp. 317-325, 2011.

[40] Y. Wu, F. Song, Y. Li et al., "Acacetin exerts antioxidant potential against atherosclerosis through Nrf2 pathway in apoE -/- Mice," Journal of Cellular and Molecular Medicine, vol. 25, no. 1, pp. 521-534, 2021.

[41] A. Liu, Q. Wu, J. Guo et al., "Statins: adverse reactions, oxidative stress and metabolic interactions," Pharmacology \& Therapeutics, vol. 195, pp. 54-84, 2019.

[42] K. B. Wallace, V. A. Sardão, and P. J. Oliveira, "Mitochondrial determinants of doxorubicin-induced cardiomyopathy," Circulation Research, vol. 126, no. 7, pp. 926-941, 2020.

[43] M. A. Mitry and J. G. Edwards, "Doxorubicin induced heart failure: phenotype and molecular mechanisms," IJC Heart \& Vasculature, vol. 10, pp. 17-24, 2016.

[44] P. Vejpongsa and E. T. H. Yeh, "Prevention of anthracyclineinduced cardiotoxicity," Journal of the American College of Cardiology, vol. 64, no. 9, pp. 938-945, 2014.

[45] Y. Octavia, C. G. Tocchetti, K. L. Gabrielson, S. Janssens, H. J. Crijns, and A. L. Moens, "Doxorubicin-induced cardiomyopathy: from molecular mechanisms to therapeutic strategies," Journal of Molecular and Cellular Cardiology, vol. 52, no. 6, pp. 1213-1225, 2012.

[46] W. Y. Wu, Y. K. Cui, Y. X. Hong et al., "Doxorubicin cardiomyopathy is ameliorated by acacetin via Sirt1-mediated activation of AMPK/Nrf2 signal molecules," Journal of Cellular and Molecular Medicine, vol. 24, no. 20, pp. 12141-12153, 2020.

[47] X. Tang, P.-H. Li, and H.-Z. Chen, "Cardiomyocyte senescence and cellular communications within myocardial microenvironments," Frontiers in Endocrinology, vol. 11, p. 280, 2020.

[48] E. J. Lesnefsky, Q. Chen, and C. L. Hoppel, "Mitochondrial metabolism in aging heart," Circulation Research, vol. 118, no. 10, pp. 1593-1611, 2016. 
[49] Y.-X. Hong, W.-Y. Wu, F. Song, C. Wu, G.-R. Li, and $\mathrm{Y}$. Wang, "Cardiac senescence is alleviated by the natural flavone acacetin via enhancing mitophagy," Aging, vol. 13, no. 12, pp. 16381-16403, 2021.

[50] S. Kumar and A. K. Pandey, "Chemistry and biological activities of flavonoids: an overview," The Scientific World Journal, vol. 2013, Article ID 162750, 16 pages, 2013.

[51] S.-D. Clas, R. I. Sanchez, and R. Nofsinger, "Chemistry-enabled drug delivery (prodrugs): recent progress and challenges," Drug Discovery Today, vol. 19, no. 1, pp. 79-87, 2014.

[52] J. Rautio, H. Kumpulainen, T. Heimbach et al., "Prodrugs: design and clinical applications," Nature Reviews Drug Discovery, vol. 7, no. 3, pp. 255-270, 2008.

[53] A. O. Basile, A. Yahi, and N. P. Tatonetti, "Artificial intelligence for drug toxicity and safety," Trends in Pharmacological Sciences, vol. 40, no. 9, pp. 624-635, 2019.

[54] A. X. Lin, G. Chan, Y. Hu et al., "Internationalization of traditional Chinese medicine: current international market, internationalization challenges and prospective suggestions," Chinese Medicine, vol. 13, no. 1, p. 9, 2018. 